\title{
Structure and Interactions of Lipid Bilayers: Role of Fluctuations
}

John F. Nagle and Stephanie Tristram-Nagle

\section{Introduction}

The cell is the fundamental unit in biology. Each cell is spatially defined by its cytoplasmic membrane. The structural basis for each membrane is lipid in bilayer form. Following this reductionist point of view, it is therefore not surprising that lipid bilayers have been much studied using a great variety of techniques.

What is surprising is the large uncertainty for simple structural quantities that has been generated by the different studies. Let us consider the most studied of all bilayers, the one composed of the lipid DPPC in the fully hydrated, biologically relevant phase ( $L_{\alpha}=$ fluid(F)=liquid-crystalline) at $T=50^{\circ} \mathrm{C}$. Various diffraction and NMR studies have given values for the interfacial area $A$ per DPPC lipid that range from $56 \AA^{2}$ to $72 \AA^{2}$ [1,2]. A most distinguished neutron diffraction study suggested $A=58 \AA^{2}$ [3] while a much used x-ray method obtained $A=71 \AA^{2}$ [4]. The real uncertainty in these numbers is even larger than the nominal $24 \%$ obtained by dividing one result by the other because $A$ for DPPC in the low temperature gel $(G)$ phase is $A_{D P P C}^{G}=48 \AA^{2}[1,5,6]$. Therefore, the effect of fluidizing the DPPC bilayer (i.e., making it biologically relevant) should be defined to be $A^{F}-A^{G}$. Using the above differences for the fluid phase area of DPPC yields an enormous uncertainty in $A_{D P P C}^{F}-A_{D P P C}^{G}$ - from $8 \AA^{2}$ to $24 \AA^{2}$. Even though one does expect to achieve as good precision in biophysics as in the physical sciences, this $100 \%$ level of uncertainty is ridiculous!

Uncertainty in $A$ is directly related to uncertainty in the bilayer thickness. A common definition of bilayer thickness is $D_{B}=2 V_{L} / A$, where $V_{L}$ is the volume of a lipid molecule in the bilayer; $V_{L}$ has been measured accurately $(0.2 \%)$ by a number of groups [7]. The thickness of lipid bilayers (vide infra for discussion of various definitions of thickness) is an important structural quantity for discussing the incorporation of intrinsic membrane proteins.

Molecular dynamics simulations give much insight into lipid bilayer structure at a level of detail not available experimentally, but uncertainty in $A$ negatively impacts such simulations. Some simulations are done with lipids and water in a simulation box of fixed size, in which case $A$ is fixed in the simulation. Results obtained from such simulations performed at the wrong $A$ 
will be misleading and could even lead the unwary simulator to vary interaction parameters in order to fit other data, such as the NMR order parameters. Many simulations are now done with constant lateral pressure [8-10]. It is then in principle possible for the simulation to find the correct value of $A$, but the computer time necessary to equilibrate can be large if the starting $A$ is far from the equilibrated value. Furthermore, even if the simulation can be equilibrated, experimental uncertainty in $A$ reduces the ability to test the interaction parameters used in the simulation.

This chapter will review some of the efforts to obtain structural results for $L_{\alpha}$ phase lipid bilayers with an emphasis on recent work from our lab. To obtain some of these structural results we found that it was necessary to deal with the effects of fluctuations. This in turn led us to the issue of interactions between bilayers, which is the second topic that will be discussed in this chapter. Underlying both these efforts is the central role of fluctuations.

Fluctuations are important in biology. The fact that the biologically relevant fluid phase of lipid bilayers is the one with the largest fluctuations supports this paradigm. Bilayers with greater fluidity can seal leaks and tears more readily. Local fluctuations in the lipid molecules affect passive permeability of solutes through the membrane and can facilitate the function of intrinsic membrane proteins by transiently reducing activation energy barriers [11]. In addition to fluctuations at the molecular length scale, there are also longer length scale fluctuations that can be adaptive for cell shape changes. Longer wavelength fluctuations give rise to an additional force between membranes, and these are the fluctuations that degrade diffraction data necessary to obtain structure. This chapter will focus upon these longer length scale fluctuations.

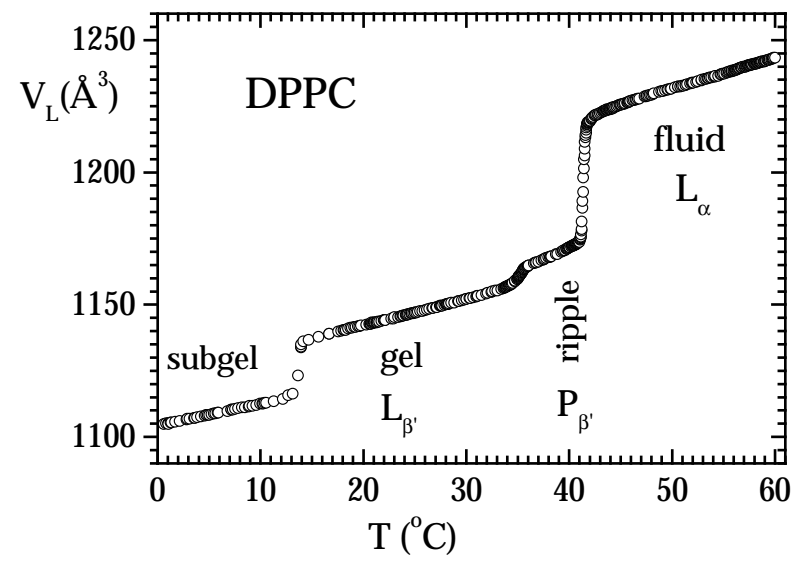

Fig. 1. Temperature dependence of lipid volume $V_{L}$ 
We will finish this general introduction with Fig. 1 which shows one key piece of structural information from our lab [12,13], the volume per lipid $V_{L}$. The temperature dependence in Fig. 1 indicates the various thermodynamic phases of DPPC. This chapter will focus on the most biologically relevant phase, identified in diffraction studies as the $L_{\alpha}$ phase. There will be some use made of results for the gel phase, which we believe is the best characterized of all the phases [5]. The chapter of Katsaras and Raghunathan [14] complements this chapter by focussing on the lower temperature phases, especially the subgel and the ripple phases.

\section{Structure}

\subsection{Levels of Description of Structure}

It is important to appreciate that it makes no sense to contemplate an atomic level structure at the sub- $\AA$ level for lipid bilayers. This is not because of poor diffraction technique or sample preparation. Lipid bilayers have biologically vital fluctuations. This means that atoms are not inherently localized. The proper description for the positions of atoms in the lipid molecule is that of broad statistical distribution functions. Fig. 2a shows simulations for distribution functions for several of the component groups of the lipid molecule along the direction of the bilayer normal [15]. The widths in this direction are of order $5 \AA$. In contrast, in the 'in-plane' direction the distribution functions for the $L_{\alpha}$ phase are just constants because the lipid molecules are in a twodimensional fluid phase. (Of course, one can still consider pair correlation functions, which are important for diffuse wide angle scattering, but this is a little explored area.) In contrast, for the lower temperature phases there is interesting and valuable in-plane structure [14,5].

Fluctuations in biologically relevant fully hydrated fluid phase bilayers mean that x-ray diffraction data can only yield electron density profiles like the one shown in Fig. 2b. The peaks in such electron density profiles are associated with the electron dense phosphate group and the lower electron density in the center is associated with the hydrocarbon region and especially with the low electron density of terminal methyl groups of the fatty acids. Therefore, electron density profiles confirm the usual picture of bilayer structure and they give another measure of the bilayer thickness, namely, the headhead thickness, $D_{H H}$. However, electron density profiles do not yield the $z$ coordinate of molecular groups along the bilayer normal. Such information has been obtained using neutron diffraction, either with selective deuteration of various component groups (DPPC at $98 \% \mathrm{RH}[3]$ ), or combined with x-ray diffraction (DOPC at $67 \% \mathrm{RH}[16]$ ).

The transverse description of the bilayer as a set of distribution functions along the $z$ axis is valuable, but it does not include other important information, such as $A$ in the lateral direction, or volumes. Therefore, a complementary description of bilayer structure, shown in Fig. 2c, is appropriate 
(a)

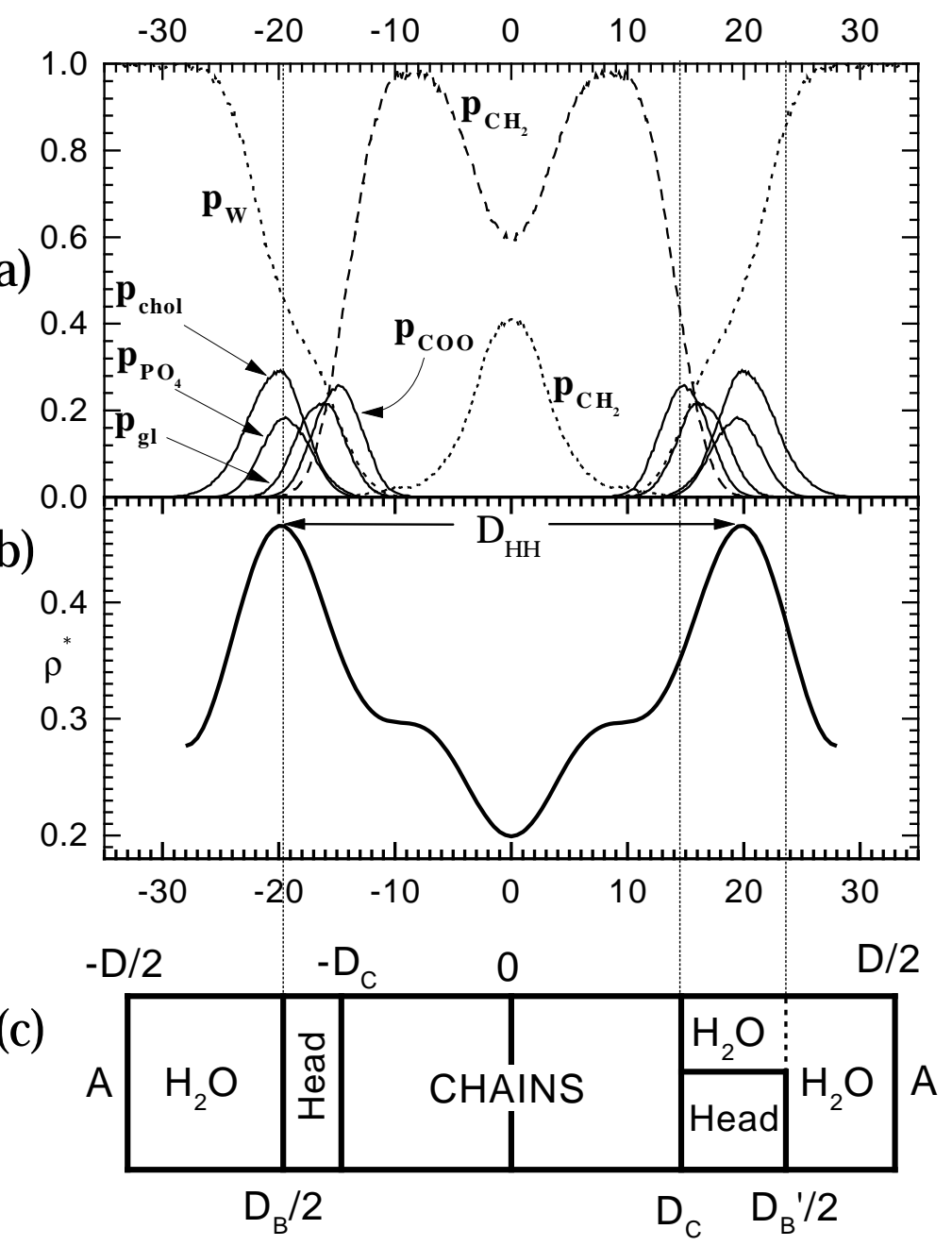

Fig. 2. Three views of bilayer structure. (a) shows probability distribution functions $p$ for different component groups. (b) shows an electron density profile. (c) shows the volumetric picture. All figures are for DPPC in the $L_{\alpha}$ phase and x-axis is in $\AA$ along the bilayer normal with same scale for (a), (b) and (c). 
[7]. The simplest description on the left of Fig. $2 c$ divides the volume $V_{L}$ of the lipid into two regions. The tail region is essentially a hydrophobic hydrocarbon chain region; by definition, it includes only the methylenes and terminal methyls on the fatty acid chains. The head region is essentially a hydrophilic region, which includes the remainder of the lipid molecule (carbonyls, glycerol, phosphate and choline). An average structure is depicted by drawing two sharp boundaries, one between chains and heads and one between heads and water, as shown on the left side of Fig. 2c. In view of the fluctuations shown in Fig. 2a, such sharp boundaries with all the chains on one side and the heads on the other are clearly artificial, but it is still a valid representation in the sense that the sharp lines can be justified as Gibbs dividing surfaces [17]. Nevertheless, in the case of the interface between the headgroups and the water, it is useful to consider a refinement to the simple description on the left side of Fig. 2c. This refinement, shown on the right side of Fig. 2c, explicitly mixes the heads and water in the polar, interfacial region. This gives better correspondence with the distribution function description in Fig. 2a; in particular, it gives a better representation of the steric thickness, defined to be $D_{B}^{\prime}$.

\subsection{Problem with the Gravimetric Method}

A popular method for obtaining structural information $[4,6]$ is most easily explained from the description shown on the left side of Fig. 2c. The total volume $V_{L}$ of one lipid molecule and its associated $n_{w}$ water molecules is $A D / 2$, where $D$ is the repeat distance that is easily and accurately measured by diffraction on stacks of lipid bilayers. (Using synchrotron x-rays and a high resolution setup, we have measured $D$ with accuracy of $0.01 \AA$, though reproducibility with nominally identical samples is usually not so good.) Therefore,

$$
A D=2\left(V_{L}+n_{w} V_{w}\right),
$$

where $V_{L}$ is the measured lipid volume [12], $V_{W}$ is the volume of water and $n_{w}$ is the number of water molecules/per lipid. The gravimetric method simply weighs the amount of water and the amount of lipid to obtain $n_{w}$. Then, $A$ is obtained as a function of $n_{w}$ from Eq. 1. The procedure is then to vary $n_{w}$ and measure $D$. In principle, as $n_{w}$ increases towards full hydration, $D$ increases until an excess water phase forms at the fully hydrated value of $n_{w}$. Increasing $n_{w}$ further just adds to the excess water phase and $D$ should remain constant.

While the concept of the gravimetric method is simple and elegant, it has been criticized and a number of studies have obtained different results $[1,18-21]$. So let us elucidate the flaw. The gravimetric method assumes that all the water added to the system goes between the lipid bilayers that are neatly stacked in regular one-dimensional arrays. In fact, gravimetric experiments are performed on lipid dispersions consisting of multilamellar vesicles 
(MLVs). Such samples have many defect regions. For example, it is customary to visualize MLVs as consisting of spheres of about $10 \mu \mathrm{m}$ diameter composed of stacks of nearly a thousand bilayers. As is well known, packing of spheres leaves defect volumes between the spheres that amount to about $26 \%$ of the total volume (for a nice schematic see Fig. 3 in [21]). Such defect volumes, which must be filled with water, escape detection by diffraction, which focusses on the more ordered structure. Therefore, the value of $n_{w}$ that should be used in Eq. 1 should be smaller than the gravimetric value of $n_{w}$ because the total weighed water includes defect water that is invisible to diffraction.

This artifact suggests that the gravimetric method will tend to overestimate $A$. Direct verification of this tendency for the gravimetric method to overestimate $n_{w}$ and $A$ was given for the gel phase of DPPC, for which inplane chain-packing and tilt angle were measured directly from wide angle diffraction. This gave $A_{D P P C}^{G}=48 \AA^{2}$ and $n_{w}=12[1,5]$. The results of the most recent gravimetric studies $[4,22]$ gave $n_{w}$ in the range $17.5-19$ which would require $A_{D P P C}^{G}$ to be in the range $52-54 \AA^{2}$ [1]. The only exception we know to this tendency of the gravimetric method to overestimate is for EPC where [23] obtained $A_{E P C}^{F}=64 \AA^{2}$ using the gravimetric method which is smaller than our best value of $A_{E P C}^{F}=69.4 \AA^{2}$ [24].

The gravimetric method also indicated that $A$ increases strongly as the limit of full hydration is approached [6,21]. Indeed, A should increase in this limit. Recall that less than full hydration is equivalent to exerting osmotic pressure $P$ on the water. The major effect of osmotic pressure is to decrease the water space $D_{w}$ and thereby the $D$ space. However, osmotic pressure also decreases $A$ because this too extracts water from stacks of bilayers. The appropriate formula to describe this second effect is [25]

$$
A=A_{0}-A D_{w} P / K_{A},
$$

where $A_{0}$ is the fully hydrated area when $P=0$ and $K_{A}$ is the phenomenological area modulus. However, while $A$ should increase as full hydration is approached, Rand and Parsegian [25] realized that the changes in $A$ obtained from the unadulterated gravimetric method became much too large near full hydration for the measured values of $K_{A}$ [26]. They then used gravimetric values of $A$ obtained under osmotic pressure at 10 atmospheres and they used Eq. 2 to extrapolate to fully hydrated $P=0$. This reduced the estimate of $A_{D P P C}^{F}$ from $71 \AA^{2}$ obtained from the unadulterated gravimetric method to $68.1 \AA^{2}$ [25]. However, this is still larger than the value obtained by an alternative method that we now proceed to discuss. 


\subsection{Electron Density Profile Method}

The electron density profile $\rho(z)$ for symmetric bilayers with a lamellar repeat spacing $D$ is

$$
\rho^{*}(z)-\rho_{W}^{*}=\frac{1}{D} F(0)+\frac{2}{D} \sum_{h=1}^{h_{\text {max }}} \alpha_{h} F_{h} \cos \left(\frac{2 \pi h z}{D}\right),
$$

where for the different orders $h>0, \alpha_{h}$ is the phase factor which can only assume values of +1 or -1 . $F_{h}$ is the bilayer form factor which is routinely obtained from the intensity $I_{h}=F_{h}^{2} / C_{h}$ under the diffraction peak. $C_{h}$ is the Lorentz polarization correction factor; for low angle scattering $C_{h}$ is nearly proportional to $h^{2}$ for unoriented MLV samples and to $h$ for oriented samples. The zeroth order form factor $F(0)$ is given by [27]

$$
A F(0)=2\left(n_{L}^{*}-\rho_{W}^{*} V_{L}\right)=2\left(\rho_{L}^{*}-\rho_{W}^{*}\right) V_{L},
$$

where $A$ is the area per lipid, $n_{L}^{*}$ is the number of electrons in the lipid molecule, $V_{L}$ is the lipid molecular volume and $\rho_{L}^{*} \equiv n_{L}^{*} / V_{L}$ is the average electron density of the lipid molecule. The form factors $F_{h}$ involve an unknown scale factor, so only the absolute ratio $r_{h}=\left|F_{h} / F_{1}\right|$ of form factors are measured directly and this means that only relative electron density profiles are routinely reported. Obtaining absolute electron density profiles will be discussed in Sec. 2.7.

The most reliable quantitative information that can be obtained from the electron density profile is the headgroup spacing $D_{H H}$, defined to be the distance between the two peaks in the electron density profile. In practice, four orders $\left(h_{\max }=4\right)$ suffice to give a reasonably accurate estimate of $D_{H H}$. Recently, we have found that, even with four orders, the measured $D_{H H}$ should be corrected due to the limited number of Fourier terms [24,28,29]. This realization came by examining reasonable model electron density profiles. The model we prefer [30], and that adequately represents the results of several simulations [31], employs a Gaussian function for the headgroup region and a Gaussian function for the terminal methyls on the chains as well as a constant for the methylene region. When we Fourier analyze this hybrid model and compare $D_{H H}$ obtained from the 4th order Fourier reconstruction with the model $D_{H H}$, we find that there is a small error that systematically varies with $D_{H H} / D[5]$. In our current use of this method (Yufeng Liu, unpublished), the parameters used to construct the hybrid model are obtained from the experimental form factors and so the hybrid model used for corrections to $D_{H H}$ is tuned to the particular lipid being studied.

McIntosh and Simon [32] introduced a method to use $D_{H H}$ to obtain $A$ for the $L_{\alpha}$ phase. The idea is to compare the more poorly determined $L_{\alpha}$ phase with the much better determined gel phase and to use differences to extrapolate from the gel phase structure to the $L_{\alpha}$ structure. Then, the $L_{\alpha}$ phase area $A^{F}$ is obtained in terms of the difference in bilayer thickness 
$\Delta D_{H H}=D_{H H}^{F}-D_{H H}^{G}$, the measured lipid volume $V_{L}^{F}$ and gel phase values for the hydrocarbon thickness $D_{C}^{G}$ and headgroup volume $V_{H}^{G}$,

$$
A^{F}=\frac{V_{L}^{F}-V_{H}^{G}}{D_{C}^{G}+\Delta D_{H H} / 2} .
$$

This method was first applied to DLPE [32] which was a favorable first choice for two reasons. The chains in DLPE are perpendicular to the bilayer in the gel phase, so gel phase quantities are easier to obtain than for PCs where the chains are tilted. However, we have been able to obtain a structural determination of gel phase DPPC in the sense of Fig. 2c [1,5]. The second reason DLPE was more favorable than the PCs is that there were four orders of diffraction for fully hydrated $L_{\alpha}$ phase DLPE, but not for DPPC, and we now turn to this major hurdle.

\subsection{Why are there so few orders of diffraction?}

The immediate shortcoming of the electron density profile approach is that fully hydrated samples of many lipids, such as unoriented DPPC dispersions in the $L_{\alpha}$ phase, have only two robust orders of diffraction. Electron density profiles using two orders of diffraction are not quantitative, even for $D_{H H}$. The generic explanation for so few orders is that fluctuations and disorder reduce higher order intensities. However, to make sense of diffraction data, it is necessary to understand that there are two quite different pieces to this explanation.

Most of the analyses of electron density and neutron scattering length profiles implicitly make the assumption that stacks of bilayers are one dimensional crystals with regular $D$ spacing. Disorder and local molecular fluctuations within each bilayer give rise to the broad distribution functions in Fig. 2a. Broad electron distribution functions, in turn, require that higher order terms in the Fourier expansion be small, so the higher order peak intensities are small. This point, which has been made forcefully by Wiener and White [33], is, however, only the first part of the explanation for the absence of higher order peaks.

The second reason for the absence of higher order peaks is that stacks of lipid bilayers are not one dimensional crystals, but smectic liquid crystals. Smectic liquid crystals have large scale (long wavelength) fluctuations that destroy crystalline long range order and replace it with quasi-long-range-order (QLRO) in which pair correlation functions diverge logarithmically instead of remaining bounded as in crystals. Because long range order is destroyed, Debye-Waller theory of scattering from crystals with fluctuations is not appropriate (see appendix to [31]). Instead, QLRO changes the scattering peak shape from an intrinsic delta function by removing intensity from the central scattering peak and spreading it into tails of diffuse scattering centered on the original peaks. The magnitude of this shifting of intensity increases with 
increasing diffraction order. For high enough order, the scattering peaks are completely converted to diffuse scattering even if the Fourier component for the local lipid bilayer is large.

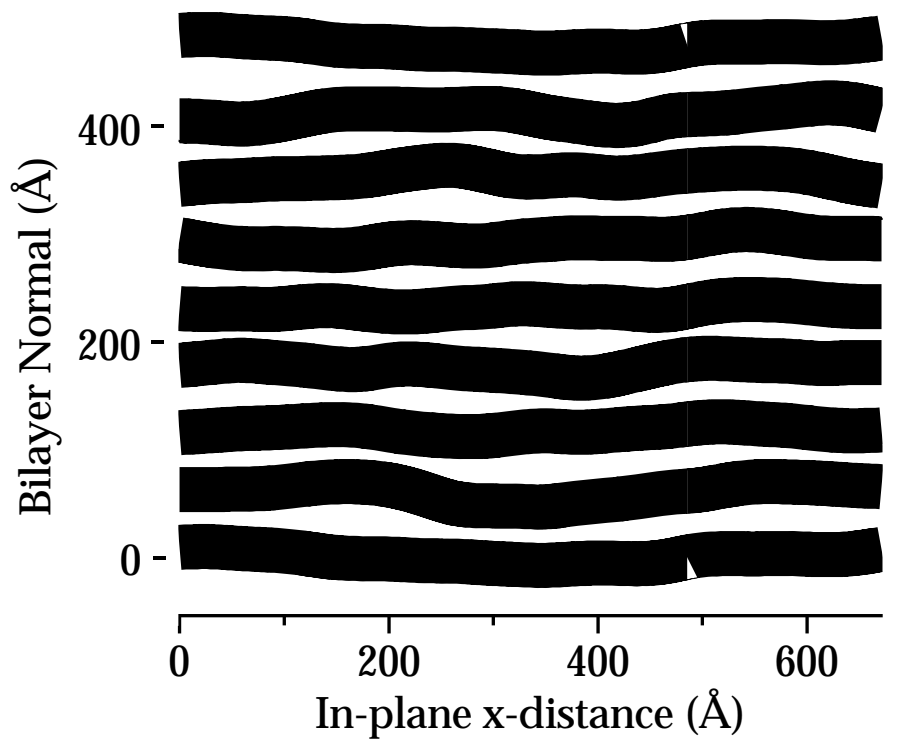

Fig. 3. Snapshot of fluctuations from a simulation [34].

The preceding distinction between short range and long range fluctuations can be summarized as follows. Short range fluctuations are intrinsic to the single lipid bilayer. These are the fluctuations that one sees in MD simulations. They correspond to disorder within a unit cell in a crystalline stack of repeat units. In contrast, long range fluctuations are fluctuations in the relative positions of the unit cells which may be thought of as the centers of the bilayer. These longer range fluctuations do not change the distribution functions of molecular components relative to the bilayer center, so they do not affect the structure of the single lipid bilayer.

Both kinds of fluctuations reduce the intensity of the higher orders. The first kind of fluctuations are local and their reduction in higher orders faithfully reflects the true bilayer structure. In contrast, the reduction in intensity due to the second kind of fluctuations at large length scale is an artifact that should be removed in order to obtain bilayer structure. This removal requires taking data with high instrumental resolution and then analyzing it using liquid crystal theory. 
A very appropriate name for this method is "liquid crystallography". This name, however, should not be confused with the same name that has been used by Wiener and White [16] in a series of papers that introduced a different major innovation, namely, the joint refinement method for combined x-ray and neutron diffraction data. Wiener and White properly emphasized that the first kind of molecular fluctuations within each unit cell are intrinsic to liquid crystals. However, this first kind of short range disorder is also present in highly disordered solids and no particular properties of liquid crystals appear in the Wiener and White analysis. It is the second kind of long range fluctuations that requires an analysis specifically tailored to liquid crystals and that we suggest should be called "liquid crystallography".

\subsection{Liquid Crystallography}

The beginning of liquid crystallography was a remarkably succinct three page paper by Caille [35] and communicated to the French Academy of Sciences by Guinier. That paper predicted power law tails for smectic liquid crystals (including stacks of bilayers) and it related the powers (exponents) to bulk phenomenological material properties, the bending modulus $K_{c}$ and the bulk compression modulus $B$; the latter represents the interactions between adjacent bilayers in a stack. These predictions of the theory were later verified by highly precise experiments on general smectics [36] and later on lipid bilayers [37].

Readers of Guinier's fine book on diffraction [38] will recall that, before Caille's paper, Guinier had discussed the important distinction between disorder of the first and second kind, where disorder of the second kind destroys crystalline long range order. Applied to a one-dimensional stack of bilayers, Guinier's theory is the same as the paracrystalline theory of Hosemann [39]. The Caille theory also treats fluctuations of the second kind, but it is considerably different from the earlier theories [38,39]. The earlier theories assumed that any disorder in the unit cell dimension propagated uniformly in the in-plane direction. This is clearly artificial because bilayers can also undulate so the local water spacing can vary with in-plane coordinates $(\mathrm{x}, \mathrm{y})$. Another major distinction between the theories is that Caille's is based on a realistic Hamiltonian model rather than the purely stochastic approach of paracrystalline theory. However, the Caille theory is considerably more difficult to apply, and paracrystalline theory has been used in biophysics, so it was necessary to test whether Caillé theory really represents a significant improvement. Our group has documented the definite superiority of Caillé theory for $L_{\alpha}$ phase DPPC bilayers [40]. On the other hand, for low temperature phases with smaller undulation fluctuations, we have found that the scattering peaks are broader and appear not to follow the Caillé form, but perhaps are dominated by frozen-in defects.

There are two main effects of liquid crystallography. The first is that the proportion of diffuse scattering to total scattering increases with order $h$. 
Indeed, for high enough $h$ the scattering is entirely diffuse and no central peak can be seen. The second is that the proportion of the scattering that is diffuse increases for all orders as the lipids become more fully hydrated.

Before the effect of fluctuations was understood, it was well known that more orders of diffraction could be obtained by drying lipid bilayer stacks. For example, Wiener and White [16] obtained $\mathrm{h}=8$ orders of diffraction for DOPC at $67 \%$ relative humidity. However, drying the sample raises the spectre that the bilayer parameters one wishes to measure are changed. Indeed, they found $A_{D O P C}^{F 67}=59.4 \AA^{2}$ for DOPC [16] whereas we find $A_{D O P C}^{F 100}=72.2 \AA^{2}$ for fully hydrated DOPC at $100 \%$ RH [29]. As mentioned in Sec. 2.2, the unadulterated gravimetric method generally gives quite large increases in $A$ near full hydration. The method of electron density profiles would seem to agree that there were large structural changes if one interprets the data from a purely crystallographic viewpoint. The higher orders of diffraction disappear and even the second order of diffraction for DPPC systematically falls off the continuous transform obtained at $98 \% \mathrm{RH}$ as the humidity is increased to full hydration [31]. However, liquid crystallography predicts these very same effects, at least qualitatively.

To verify that liquid crystallography predictions are quantitative requires considerably more effort. The first effort, skillfully carried out by R. Zhang in our lab, was to improve the Caille theory to give quantitative predictions, not just for the power laws, but also for the amplitudes of the scattering [41]. The ensuing modified Caillé theory enables us to predict the shapes of the scattering peaks for all orders using only a few parameters, primarily the average domain size $L$, which affects the width of the central peak, and the Caillé $\eta_{1}$ parameter [35],

$$
\eta_{1}=\frac{k_{B} T}{8 \sqrt{B K_{c}}} \frac{4 \pi}{D^{2}}
$$

$\eta_{1}$ is proportional to the mean square fluctuations $\sigma^{2}$ in the water space and $\eta_{1}$ governs the size of the scattering tails as well as the power law decay. The second effort was to obtain the peak shapes experimentally. We use a silicon analyser crystal with instrumental resolution $\Delta q=0.0001 \AA^{-1}$ [40]. However, with such high resolution, most scattered x-rays do not get to the detector, so a synchrotron source is helpful and we use the CHESS facility at Cornell. We are able to measure sufficiently far into the power law tails (before signal/noise becomes too small) so that we can obtain the $\eta_{1}$ parameter. It might be noted that the classic way of obtaining power law exponents such as $\eta_{1}$ is to use $\log -\log$ plots $[36,37]$. This is difficult because the number of decades in $\Delta q$ over which straight line behavior on a log-log plot can be seen is rather small. The small $\Delta q$ range in the central peak is limited by the crossover to a regime dominated by the sample domain/correlation size $L$ and the large $\Delta q$ range is limited by signal/noise and is further degraded by continuous changes in the form factor $F(q)$. In contrast, our method relies, 
not only on the power law behavior, but also on the larger amplitudes in the tails when $\eta_{1}$ is larger. Once we have obtained the parameters in the model, we can extrapolate the diffuse scattering that is in the tails of the structure factor $S(q)$. Even though this extrapolated diffuse scattering intensity is so small that it can't be separated from background, the total amount of it is large because it extends all the way between scattering peaks. Fig. 4 indicates the amount of integrated intensity that is recovered using this extrapolation. When this missing intensity is added, the result is that liquid crystallography does indeed predict the effects in the preceding paragraph quantitatively, and the use of it enables more accurate form factors $F_{h}$ to be obtained that are true to the bilayer structure. Our former student, Horia Petrache, has made available a program to perform liquid crystallography data analysis. (Send e-mail to nagle@andrew.cmu.edu to obtain access.)

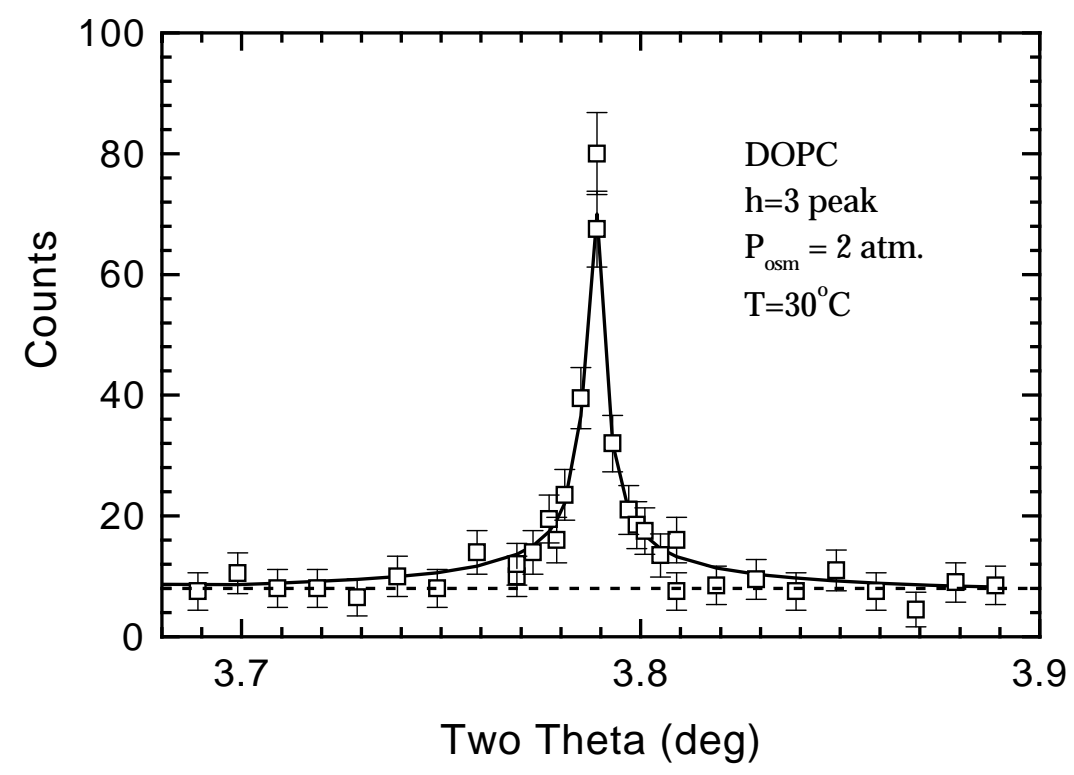

Fig. 4. Example of missing diffuse scattering under $h=3$ peak in DOPC. The solid line is the fit that is determined by the first three orders. If one uses the dashed line as a baseline, one would miss the intensity under the dashed line in the figure as well as the intensity under the continuation (halfway to the next order) of the solid lines outside the figure. 


\subsection{Structural Results}

The method we have been using to obtain structural results first obtains fluctuation corrected form factors for unoriented samples using liquid crystallography. Electron density profiles are drawn for those samples that have four orders of diffraction. Such samples are typically under osmotic stress of 20-60 atmospheres, corresponding to relative humidities of $95-98 \%$. To exert osmotic pressure we use the now classic method of Rand and Parsegian [25] with the polymer PVP or dextran. There is never a problem of choosing the correct phases for $F_{h}$; either plots of $F(q)$ at many osmotic pressures or fitting hybrid electron density models to the intensities always give unambiguous phases, which are often $(--+-)$. For PC lipids, we use Eq. 5 to obtain $A$ as a function of osmotic pressure $P$. The reference phase that we have used in Eq. 5 is the gel phase of DPPC, for which headgroup volume $V_{H}^{G}$ and hydrocarbon thickness $D_{C}^{G}$ are accurately known from gel phase studies $[1,5]$. The volume $V_{L}^{F}$ in the $L_{\alpha}$ phase is accurately $( \pm 0.2 \%)$ measured [12]. The value of $\Delta D_{H H}$ used in Eq. 5 is obtained from the electron density profiles. Inspired by Eq. 2, we plot the ensuing values of $A$ against $A D_{w} P$, where the slope is $-1 / K_{A}$ and the intercept is the full hydration value $A_{o}$. To do this we also need the Luzzati water thickness $D_{w}$, which is obtained from the partitioning indicated in Eq. 1, namely, $A D_{w}=n_{w} V_{w}$, where $n_{w}$ is now obtained directly from Eq. 1 and the value of $A$. (Note that Eq. 1 is valid, even if the gravimetric method of using it is not.)

Fig. 5 shows $A$ vs. $A D_{w} P$ for DMPC and EPC [24]. Because there are only three data points for each lipid, fits with both slope $-1 / K_{A}$ and intercept $A_{0}$ unconstrained give large uncertainties in the slopes, $K_{A}=81-201$ dyn $/ \mathrm{cm}$ for EPC, but the fractional uncertainties are much smaller for the fully hydrated area $A_{0}=69.4 \pm 1.1 \AA^{2}$. When we recently published these results, we had overlooked an independent measurement, $K_{A}=167 \pm 23$, for giant unilamellar vesicles of EPC [42]. If we now constrain the slope with this value of $K_{A}$, we obtain $A_{0}=68.6 \AA^{2}$.

In the case of DMPC, $K_{A}$ for giant unilamellar vesicles is $145 \mathrm{dyn} / \mathrm{cm}$ [26]. One concern is that this kind of measurement of $K_{A}$ is in the expansion mode whereas our use of $K_{A}$ is in the compression mode. This issue was recently addressed using NMR as a function of osmotic pressure with nearly the same result $K_{A}=141 \mathrm{dyn} / \mathrm{cm}$ [21]. This $K_{A}$ was combined with a gravimetric x-ray determination of $A$ limited to osmotic pressures greater than 20 atmospheres $(98 \% \mathrm{RH})$ to obtain $A_{0}=59.5 \AA^{2}$ [21]. In comparison, our unconstrained result $K_{A}=108 \mathrm{dyn} / \mathrm{cm}$ is again somewhat smaller than the measured values, but this makes little difference in our value of $A_{0}$. Constraining the slope $K_{A}$ gives excellent agreement of our $A_{0}=59.4 \AA^{2}$ with [21].

In the case of DOPC, our results suggested that $K_{A}$ was roughly 190 dyn $/ \mathrm{cm}$ and $A_{0}=72 \AA^{2}$ [29]. Our earlier results for DPPC [31] did not allow 


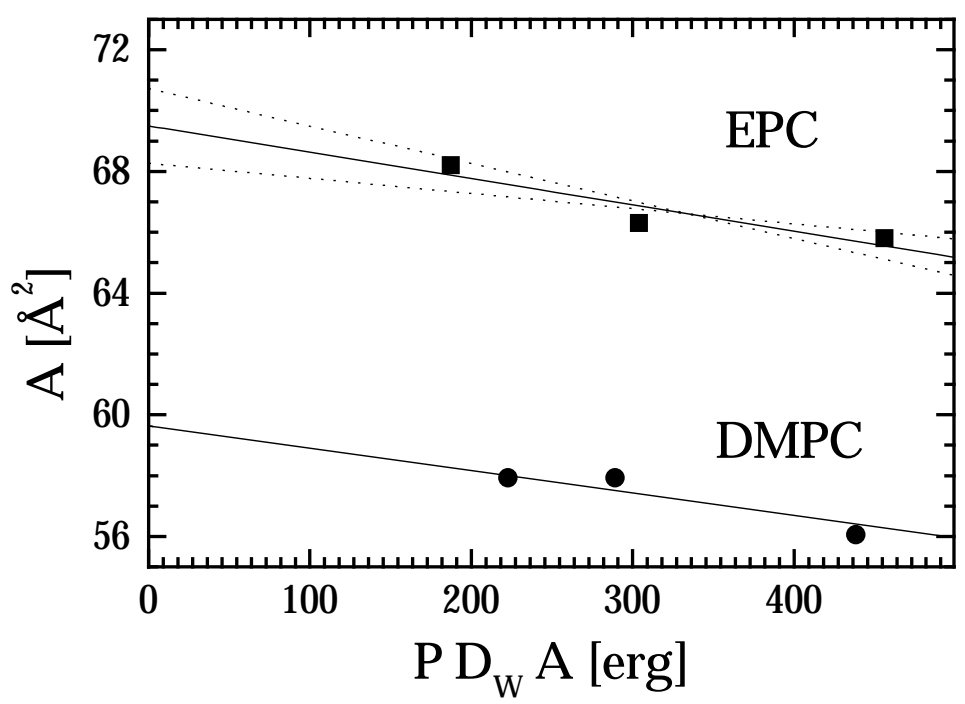

Fig. 5. Dependence of $A$ upon drying using osmotic pressure $P$ in terms of $P D_{W} A$. This makes the slope equal to $-1 / K_{A}$ and the intercept equal to $A_{\circ}$ at full hydration as seen in Eq. 2.

for a non-infinite $K_{A}$. If they had, our best $A$ would probably increase to about $64 \AA^{2}$ from our quoted value $A=62.9 \pm 1.3 \AA^{2}$ [31].

\subsection{Absolute Electron Density Profiles}

Obtaining absolute electron density profiles requires information in addition to low angle scattering. Wide angle x-ray studies of the gel phase and volumetric studies as a function of temperature give the electron density of the lipid molecule $V_{L}$ and some of its component groups, especially the methylenes $V_{\mathrm{CH}_{2}}$ and the terminal methyls $V_{\mathrm{CH}_{3}}$ in the chains [7,12]. This kind of information is better used with the hybrid electron density model [30] than with the Fourier representation. Also, the hybrid model has the additional advantage over the Fourier representation in that data for many samples, including different osmotic pressures and $D$ spacings if there is little change in structure, can be used simultaneously to obtain the best fit.

If one fits any model to measured relative form factors, the model must contain an unknown scale factor $K$. One way to constrain $K$ in the hybrid model is to require that the model have the correct value for the electron density in the methylene plateau. Another way is to require that the methyl trough be the correct size to account for the known deficit of electron density in the terminal chain methyls. Yet another way to constrain $K$ is to require 
that the model has the value of $F(0)$ that is obtained from $V_{L}$ and $A$ using Eq. 4. (This latter way cannot work when $F(0)$ is effectively zero, which is the case for many $L_{\alpha}$ phases, but even then $F(0)$ still acts as a constraint on the overall hybrid model parameters.) Although any one of these constraints should suffice in principle, in practice, when only one or two are applied, the others are then not satisfied. It is therefore best to use all three constraints simultaneously [31]. This is not surprising or disturbing because the low angle diffraction information is confined to small $q$, corresponding to $h=4$, so low angle x-ray information should be supplemented as much as possible by other information.

The preceding, somewhat strenuous, method of constructing electron density profiles has only been applied to the DPPC $L_{\alpha}$ phase [31]. It has also been applied to the $L_{\beta^{\prime}}$ phase, but with data only at full hydration [30]. Derivation of absolute electron density profiles for other PC lipids is based on a simple argument. Since the headgroups are the same, the integrated electron density under the headgroup peak in excess of the level due to water on one side, and hydrocarbon on the other, should scale inversely with the area $A$, and the prefactor can be determined from $V_{H}$ and the number of electrons in the headgroup [24].

It might also be noted that one could contemplate scaling the electron density profiles from simulations. However, different simulations give rather different scaling factors (see Fig. 7 in [31]), so a more immediate use of absolute electron density profiles is to test simulations.

\section{Interactions between Bilayers}

From the preceding section, it is clear that long range fluctuations of the second kind are really a nuisance for obtaining average structure of lipid bilayers in the highly fluctuating, fully hydrated, biologically relevant $L_{\alpha}$ phase. From a structural point of view these fluctuations have no intrinsic value. We now turn to a topic where these fluctuations do have intrinsic importance that is directly addressed by liquid crystallography.

\subsection{Hard versus Soft Confinement Regimes}

As was originally shown by Helfrich [43], long range fluctuations are the cause of an effective interaction between lipid bilayers that is called the fluctuation interaction. The conceptual basis for this interaction is that two bilayers close to one another cannot fluctuate as much as two bilayers far from each other. Mutual suppression of independent fluctuations leads to a decrease in entropy and an increase in free energy as the average water separation distance $a$ is decreased, so this interaction is repulsive and entropic. It is an entropic energy (-TS) that is absent at absolute zero temperature, rather than a bare energetic interaction (E). 
Helfrich showed that, when the only bare energetic interaction between bilayers is steric (excluded volume interaction), the form of the effective fluctuation free energy is [43]

$$
f_{U}=0.42 \frac{\left(k_{B} T\right)^{2}}{K_{c} a^{2}} .
$$

This result has been confirmed experimentally in systems where the bare interaction $V_{B}(a)$ can be closely approximated as zero over most of the relevant range in water spacing $a$ [44]. This regime is called the hard confinement regime because the bare potential can be thought of as confinement of each bilayer between hard walls formed by neighboring bilayers. Of course, the neighboring bilayers can also move, so the hard wall potential also fluctuates, but this does not change the form of the fluctuation interaction. Everything about the hard confinement regime is quite well established, except for the magnitude of the prefactor [45].

In general there are additional bare interactions besides the obvious steric interaction. If these interactions have ranges that are comparable to the average water spacing $a$, then the approximation of the bare interaction $V_{B}(a)$ by a hard box-like potential is obviously deficient. It is then appropriate to consider a soft confinement regime [46,47].

One important bare interaction is the strong repulsive hydration force which, even though not so well understood, has been well documented experimentally $[25,42]$ to have the form

$$
V_{h y d}(z)=P_{h} \lambda e^{-z / \lambda},
$$

with parameters $\lambda$ (decay length) and prefactor $P_{h}$. Another important bare interaction is the van der Waals attractive interaction,

$$
V_{v d W}(z)=-\frac{H}{12 \pi}\left(\frac{1}{z^{2}}-\frac{2}{\left(z+D_{B}\right)^{2}}+\frac{1}{\left(z+2 D_{B}\right)^{2}}\right),
$$

where $D_{B}$ is the bilayer thickness and $H$ is the Hamaker parameter. This is the interaction assumed to be responsible for limiting the swelling in bilayers composed of lipids with no net charge. We will define $a_{0}$ to be the limiting water space for fully hydrated bilayers with osmotic pressure $P=0$. Because $a_{o}$ is only $10-30 \AA$, a graph of bare potential $V_{B}(z)$ versus $z$ on this length scale shows considerable variation, especially as $z$ approaches 0 . For charged lipids in low salt, one should also consider an electrostatic interaction, but this is absent for the neutral lipids. An additional very short range repulsion has been measured and attributed to protrusions [48]. We do not include it since it only plays a role for lipids under high osmotic pressure and small water space $a$. It does play the formal role of suppressing the singularity in the van der Waals potential at $z=0$. 
It has been proposed for the soft confinement regime that the fluctuation interaction free energy in Eq. (7) should be modified [47,46] and a formula involving an exponential with decay length $\lambda_{f l}$

$$
f_{U 2}=\left(\pi k_{B} T / 16\right)\left(P_{h} / K_{c} \lambda\right)^{1 / 2} \exp \left(-a / \lambda_{f l}\right),
$$

has been offered $[26,47]$. This exponential functional form is quite different from the power law form in Eq. 7 established for the hard confinement regime. Furthermore, the decay length $\lambda_{f l}$ was predicted to be twice the decay length $2 \lambda$ of the hydration force.

For lipid bilayers the now traditional way [25] to investigate interbilayer forces experimentally is to measure the average water space $a$ as osmotic pressure $P$ is varied; such data are usually plotted as $\log P(a)$ as in Fig. 6. The data from many groups clearly show an exponential increase for $P$ greater than 10 atmospheres and this is the experimental basis for the force that is named the hydration force. However, there are at least three energies involved with four parameters $\left(\lambda, P_{h}, H\right.$ and $\left.K_{c}\right)$. There are also uncertainties in how one defines and obtains water space $a$ (gravimetric $D_{W}$ versus steric $D_{W}^{\prime}$ - see Fig. 2c) and bilayer thickness $\left(D_{B}\right.$ vs $\left.D_{B}^{\prime}\right)[7]$. While it has been encouraging that fits to the $P(a)$ data make sense with reasonable values for the parameters [49], there are too few data to provide fits that uniquely separate $P(a)$ into its constituent forces. As noted by Parsegian and Rand [17], “... dissection of the measured pressure $P$ into its physically distinct components is a problem almost as difficult as the theoretical explanation of these components themselves". In particular, the functional form of the fluctuation pressure is an important assumption in carrying out such fits. Since the derivation of the soft confinement formula for fluctuation pressure was non-trivial (involving some close self-consistency arguments that are difficult to improve upon and difficult to validate by analytic theory), it therefore seemed appropriate to test Eq. 10.

\subsection{Experimental Window on the Fluctuation Force}

We realized that our experimental study of the fluctuation correction for structural studies also provided an experimental window on fluctuational forces that could help reduce the ambiguity inherent in only using $P(a)$ data; this was a major part of Horia Petrache's research in our group. The most direct connection is that the fluctuational free energy $F_{f l}$ is related to the Caillé $\eta_{1}$ parameter [50] by

$$
F_{f l}=\left(\frac{k_{B} T}{2 D}\right)^{2} \frac{1}{K_{c} \eta_{1}} .
$$

Since the bending modulus $K_{c}$ is a property of the single, isolated bilayer, the functional form of $F_{f l}(a)$ can be obtained from $\eta_{1}$ and $D$. A plot of $1 / \eta_{1} D^{2}$ therefore shows the functional form of $F_{f l}$. Data for EPC are shown in Fig. 7 . 


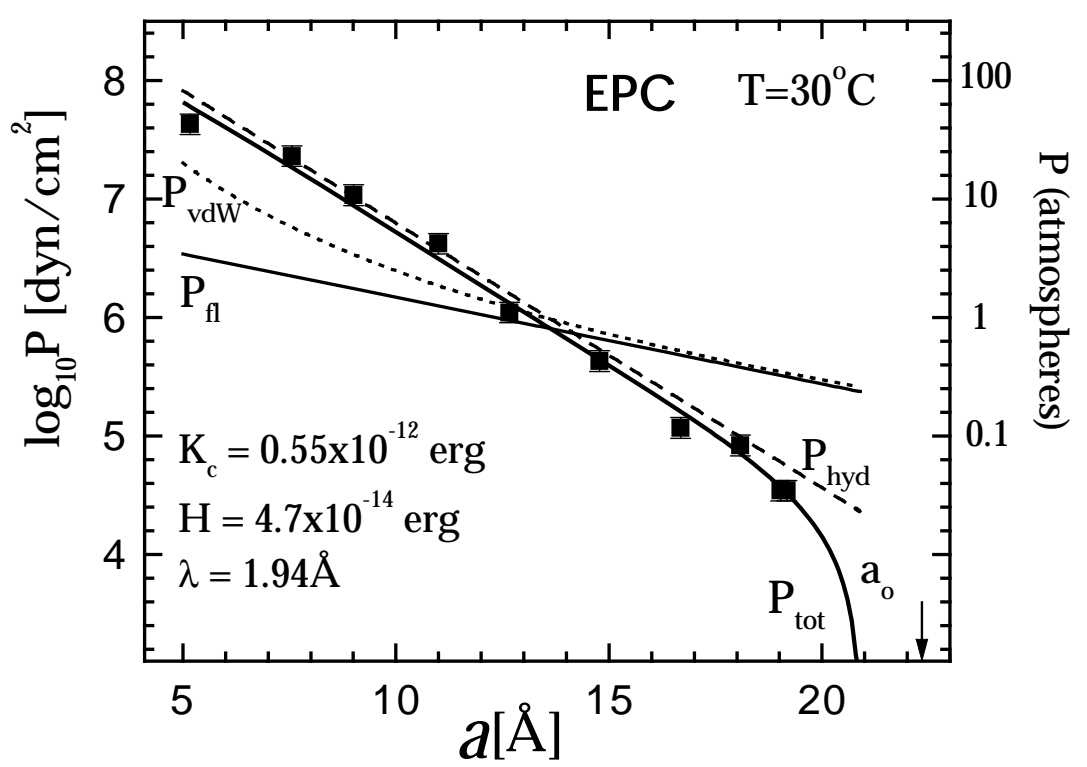

Fig. 6. Osmotic pressure $P$ versus water space $a$. Various lines show contributions from various interactions, with the bold solid curve showing the fitted total $P$.

Data for DPPC, DMPC, EPC [50] and DOPC [29] are all inconsistent with the hard confinement functional form in Eq. 7, proving that a theory of soft confinement is necessary. The data are consistent with the prediction of the soft confinement theory that the fluctuation free energy has an exponential decay with $a$. However, the effective decay length of the fluctuation free energy, which is defined to be $\lambda_{f l}$, is consistently larger than the theoretical prediction $\lambda_{f l}=2 \lambda$ as indicated in Fig. 7 for EPC. For the four lipids that we have studied, the ratio $\lambda_{f l} / \lambda$ is in the range $2.5-3$.

\subsection{Simulations}

We undertook simulations to address several questions regarding interactions. The first one, from the preceding subsection, concerns our experimental result that $\lambda_{f l} / \lambda$ is consistently greater than 2 . This result could have been due to several reasons, including: (i) the soft confinement theory (Eq. 10) may not be correct, (ii) the bare interactions may be incorrectly described or (iii) there may be experimental discrepancies. By doing a simulation with the same form of the interactions as in Eq. 8 and Eq. 9, we bypassed (ii) and (iii) and tested (i) directly. The result of the simulation is that $\lambda_{f l} / \lambda$ is about 2.4 [34]. Although this is a bit smaller than the experimental ratio, it clearly agrees 


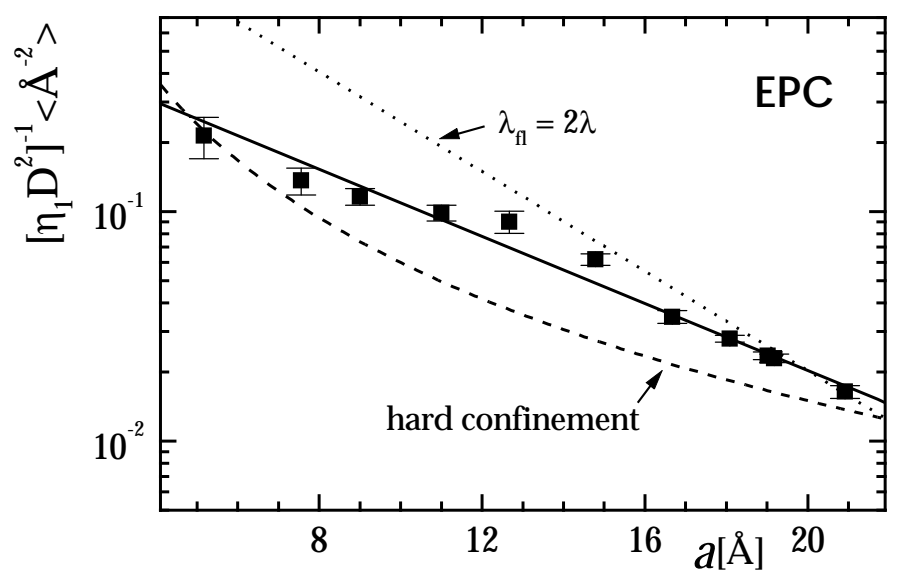

Fig. 7. Functional form of fluctuation free energy versus water spacing is represented by an exponential with decay length $\lambda_{f l}=5.9 \AA$

with the experimental conclusion that the ratio is larger than the value of 2 given by Eq. 10.

The simulations were at the nano-scale in which the bilayer was treated as an elastic continuum with a bending modulus $K_{c}$ and the interactions between bilayers consisted of the phenomenological van der Waals and hydration forces. This is the appropriate length scale for the long wavelength fluctuation forces. In particular, atomic level molecular dynamics simulations are not able to probe this longer length scale because the system size and number of atoms becomes prohibitively large to equilibrate in reasonable computer times. Since motional dynamics are unclear at the nano-scale, the appropriate kind of simulation is Monte Carlo rather than molecular dynamics. Monte Carlo also has the advantage that sampling phase space can be done more efficiently than following equations of dynamical motion. Nikolai Gouliaev in our lab implemented a particularly efficient algorithm that employed changes in the Fourier coefficients of the bilayer positions so that rather large, multiple moves in real space could be made while changing the bending energy by small amounts. This method, which we call Fourier Monte Carlo, decreases the equilibration time by a factor of at least 30 when compared to the usual method of moving one local point on the bilayer at each step [51]. This allowed Gouliaev to extrapolate thermodynamic properties to stacks consisting of many larger bilayers by using sequences consisting of as many as $M=32$ bilayers in a stack, $N^{2}=1024$ Fourier modes (equivalent to lattice sites) and lateral extents $L$ up to $2800 \AA$ [34,51]. Fig. 3 shows a snapshot of a slice through a simulation of $M=8$ bilayers. 
The thermodynamic quantities of greatest interest are the osmotic pressure $P(a)$ and the root mean square fluctuation $\sigma(a)$ in water spacing, both as a function of mean interbilayer spacing $a . \sigma$ is simply related to the measured Caillé $\eta_{1}$ parameter [50] by

$$
\sigma^{2}=\eta_{1} D^{2} / \pi^{2}
$$

The simulation results compare favorably with the analytic theory [47] for small $a$ and when there are no van der Waals interactions, but the discrepancy grows as $a$ approaches full hydration when $P=0[34,51]$. These discrepancies are too large to ignore when trying to fit data.

The simulations also allowed us to address another issue [34]. Our experimental analysis that determines the Caillé $\eta_{1}$ parameter is based on liquid crystal theory which assumes that the interaction between bilayers is harmonic. However, the phenomenological potential consisting of the sum of van der Waals and hydration interactions is not harmonic. Furthermore, the analytic theory approximates the true potential self-consistently with a harmonic potential. Since the analytic theory shows discrepancies with the results of the simulations, the obvious question was whether the fluctuational data analysis is also flawed. However, the diffraction line shapes depend upon the functional form of the pair correlation functions. The simulations show that the functional form for the true potentials agrees well with that obtained from harmonic theory, thereby validating the data analysis. The problem with the analytic theory is that the self-consistency relation gives a different value for the harmonic modulus than the one that best describes the results of the simulations [34].

\subsection{Determination of Interaction Parameters}

An important goal now is to determine the values of the interaction parameters. The basic experimental approach [50] determined the decay length $\lambda_{f l}$ of the fluctuation force and its magnitude up to a factor of the bending modulus $K_{c}$. Given a value of $K_{c}$, fits to the bare pressure $P_{b a r e}(a)=P(a)-P_{f l}$ gave well determined values for $H_{\text {Hamaker }}, \lambda$ and $P_{h}$. However, fits with different values of $K_{c}$ over the range spanned by literature values gave equally good fits, essentially because variations in $H$ compensated for variations in $K_{c}$ whereas values of $\lambda$ (about $2 \AA$ ) and $P_{h}$ were robustly determined [50].

The basic experimental approach of the preceding paragraph used the fluctuation data $\eta_{1}(a)$ only to eliminate the effective modulus $B$ for interbilayer interactions and this throws away information about the absolute size of the fluctuations. Simulations, however, give both $P(a)$ and $\eta_{1}(a)$. Requiring both to agree with the data is a stronger constraint on the interaction parameters. Detailed fits of simulations and data have not yet been carried out for all the lipids our group has worked on. However, for DMPC at $30^{\circ} \mathrm{C}$ the following parameter set fits both $P(a)$ and $\eta_{1}(a)$ fairly well [52]: $H=7.13 \cdot 10^{-14} \mathrm{erg}$, 
$K_{c}=0.5 \cdot 10^{-12} \mathrm{erg}, \lambda=1.91 \AA$ and $P_{h}=1.32 \cdot 10^{9} \mathrm{erg} / \mathrm{cm}^{2}$ and it is clear that larger values of $K_{c}$ provide inferior fits. This value of $K_{c}$ agrees well with [53] and is smaller than the value given by [54]. The value of $H$ is somewhat larger than preferred by [55]. However, more lipid systems should be studied before drawing definitive conclusions for these parameter values.

Acknowledgements: We wish to acknowledge some excellent graduate students who did much of this work. Michael Wiener originally got us involved in x-ray studies in collaboration with our colleague, Prof. Bob Suter. Ruitian Zhang made the breakthrough into true liquid crystallography. Wenjun Sun performed the studies of gel phase lipid which were necessary to complete the structures of the more biologically relevant fully hydrated $L_{\alpha}$ phase. Horia Petrache was central to our subsequent development of liquid crystallography and to getting into interbilayer interactions. Nikolai Gouliaev was also involved in the theory of interbilayer interactions and developed the Monte Carlo methods to model them. We owe a debt to communism for inadvertently sending us all but one of these students. We acknowledge CHESS for beamtime (NSF-DMR97-13424) and the help of many of the staff, especially our collaborator Dr. Randy Headrick. For support of our research we gratefully acknowledge NIH Grant GM44976. 


\section{References}

1. S. Tristram-Nagle, R. Zhang, R. M. Suter, C. R. Worthington, W.-J. Sun and J. F. Nagle: Measurement of chain tilt angle in fully hydrated bilayers of gel phase lecithins, Biophys. J. 64, 1097-1109 (1993)

2. J. F. Nagle: Area/lipid of bilayers from NMR. Biophys. J. 64, 1476-1481 (1993)

3. G. Buldt, H. U. Gally, J. Seelig, G. Zaccai: Neutron Diffraction Studies on Phosphatidylcholine Model Membranes. I. Headgroup Conformation, J. Mol. Biol. 134, 673-691 (1979). See p. 689.

4. L. J. Lis, M. McAlister, N. Fuller, R. P. Rand and V. A. Parsegian: Interactions between neutral phospholipid bilayer membranes, Biophys. J. 37, 657-666 (1982)

5. W.- J. Sun, R. M. Suter, M. A. Knewtson, C. R. Worthington, S. TristramNagle, R. Zhang and J. F. Nagle: Order and disorder in fully hydrated unoriented bilayers of gel phase dipalmitoylphosphatidylcholine, Phys. Rev. E 49, 4665-4676 (1994)

6. A. Tardieu, V. Luzzati, and F. C. Reman: Structure and polymorphism of the hydrocarbon chains of lipids; a study of lecithin-water phases, J. Mol. Biol. 75, 711-733 (1973)

7. J. F. Nagle and M. C. Wiener: Structure of fully hydrated bilayer dispersions, Biochim. Biophys. Acta 942, 1-10 (1988)

8. D. P. Tieleman, S. J. Marrink and H. J. C. Berendsen: A computer perspective of membranes: Molecular dynamics studies of lipid bilayer systems, Biochim. Biophys. Acta 1331, 235-270 (1997)

9. D. J. Tobias, K. Tu and M. L. Klein: Atomic-scale molecular dynamics simulations of lipid membranes, Current Opinion in Colloid and Interface Science 2, $15-26$ (1997)

10. A. M. Smondyrev and M. L. Berkowitz: Molecular Dynamics Simulations of DPPC Bilayers in DMSO, Biophys. J. 76, 2472-2478 (1999)

11. S. M. Gruner and E. Shyamsunder: Is the mechanism of general anesthesia related to lipid membrane spontaneous curvature?, Ann. N. Y. Acad. Sci 625, 685-691 (1991).

12. J. F. Nagle and D. A. Wilkinson: Lecithin bilayers: Density measurements and molecular interactions, Biophys. J. 23, 159-175 (1978)

13. M. C. Wiener, S. Tristram-Nagle, D. A. Wilkinson, L. E. Campbell and J. F. Nagle: Specific volumes of lipids in fully hydrated bilayer dispersions. Biochim. Biophys. Acta 938, 135-142 (1988)

14. J. Katsaras and V. A. Raghunathan, this volume

15. H. I. Petrache, S. E. Feller and J. F. Nagle: Determination of component volumes of lipid bilayers from simulations. Biophys. J. 72, 2237-2242

16. M. C. Wiener and S. H. White: Structure of a fluid dioleoylphosphatidylcholine bilayer determined by joint refinement of x-ray and netron diffraction data. III. Complete structure. Biophys. J. 61, 434-447 (1992)

17. V. A. Parsegian and R. P. Rand: Interaction in membrane assemblies, in Structure and dynamics of membranes, ed. R. Lipowsky and E. Sackmann (North Holland, Amsterdam 1995) pp. 643-690 Langmuir 11, 1047 (1995)

18. G. Klose, B. W. Koenig, H. W. Meyer, G. Schulze and G. Degovics: Small-angle $\mathrm{x}$-ray scattering and electron microscopy of crude dispersions of swelling lipids and the influence of the morphology on the repeat distance, Chem. Phys. Lipids 47 225-234 (1988) 
19. M. Kodama, H. Aoki, H. Takahashi and I. Hatta: Interlamellar waters in dimyristoylphosphatidylethanolamine-water system as studied by calorimetry and x-ray diffraction, Biochim. Biophys. Acta 1329 61-73 (1997)

20. T. J. McIntosh and S. Simon: Hydration force and bilayer deformation: A reevaluation, Biochemistry 25, 4058-4066 (1986)

21. B. W. Koenig, H. H. Strey, and K. Gawrisch: Membrane lateral compressibility determined by NMR and X-ray diffraction: effect of acyl chain polyunsaturation, Biophys. J. 73 1954-66 (1997)

22. M. J. Ruocco and G. G. Shipley: Characterization of the sub-transition of hydrated DPPC bilayers; kinetic, hydration and structural study, Biochem. Biophys. Acta 691, 309-320 (1982)

23. T. J. McIntosh, A. D. Magid and S. A. Simon: Range of the solvation pressure between lipid membranes: Dependence on the packing density of solvent molecules, Biochem. 28, 7904-7912 (1989)

24. H. I. Petrache, S. Tristram-Nagle and J. F. Nagle: Fluid phase structure of EPC and DMPC bilayers, Chem. Phys. Lipids 95, 83-94 (1998)

25. R. P. Rand and V. A. Parsegian: Hydration forces between phospholipid bilayers, Biochim. Biophys. Acta 988, 351-376 (1989)

26. E. A. Evans and D. Needham: Physical properties of surfactant bilayer membranes: Thermal transitions, elasticity, rigidity, cohesion, and colloidal interactions. J. Phys. Chem. 91, 4219-4228 (1987)

27. J. F. Nagle and M. C. Wiener: Relations for lipid bilayers: Connection of electron density profiles to other structural quantities, Biophys. J. 55, 309-313 (1989)

28. W.-J. Sun, S. Tristram-Nagle, R. M. Suter and J. F. Nagle: Structure of gel phase saturated lecithin bilayers: Temperature and chain length dependence, Biophys. J. 71, 885-891 (1996)

29. S. Tristram-Nagle, H. I. Petrache and J. F. Nagle: Structure and interactions of fully hydrated dioleoylphospatidylcholine bilayers, Biophys. J. 75, 917-925 (1998)

30. M. C. Wiener, R. M. Suter and J. F Nagle: Structure of the fully hydrated gel phase of DPPC, Bophys. J. 53, 315-325 (1989)

31. J. F. Nagle, R. Zhang, S. Tristram-Nagle, W-S. Sun, H. I. Petrache, and R. M. Suter: X-ray structure determination of fully hydrated $L_{\alpha}$ phase dipalmitoylphosphatidylcholine bilayers, Biophys. J. 70, 1419-1431 (1996)

32. T. J. McIntosh and S. A. Simon: Area per molecule and distribution of water in fully hydrated dilauroylphosphatidylethanolamine bilayers, Biochemistry $\mathbf{2 5}$, 4948-4952 (1986)

33. M. C. Wiener and S. H. White: Fluid bilayer structure determination by the combined use of X-ray and neutron diffraction I. Fluid bilayer models and the limits of resolution, Biophys. J. 59, 162-173 (1991)

34. N. Gouliaev and J. F. Nagle: Simulations of interacting membranes in the soft confinement regime, Phys. Rev. Lett. 81, 2610-2613 (1998)

35. A. Caillé: Physique cristalline - Remargues sur la diffusion des rayons X dans les smectiques A., C. R. Acad. Sc. Paris Serie B 274, 891-893 (1972)

36. J. Als-Nielsen, J. D. Litster, R. J. Birgeneau, M. Kaplan, C. R. Safinya, A. Lindegaad-Anderson, and S. Mathiesen: Observation of algebraic decay of positional order in a smectic liquid crystal, Phys. Rev. B 22, 312-320 (1980) 
37. D. Roux and C. R. Safinya: A synchrotron X-ray study of competing undulation and electrostatic interlayer interactions in fluid multimembrane lyotropic phases, J. Phys. France 49, 307-318 (1988)

38. A. Guinier: X-Ray Diffraction (W. H. Freeman and Company, San Francisco 1963) pp 300 and 304

39. R. Hosemann and S. N. Bagchi: Direct Analysis of Diffraction by Matter (NorthHolland, Amsterdam 1962)

40. R. Zhang, S. Tristram-Nagle, W.-J. Sun, R. L. Headrick, T. C. Irving, R. M. Suter and J. F. Nagle: Small-angle X-ray scattering from lipid bilayers is well described by modified Caillé theory but not by paracrystalline theory, Biophys. J. 70, 349-357 (1994)

41. R. Zhang, R. M. Suter and J. F. Nagle: Theory of the structure factor of lipid bilayers, Phys. Rev. E 50, 5047-5060 (1994).

42. T. J. McIntosh, S. Advani, R. E. Burton, D. V. Zhelev, D. Needham and S. A. Simon: Experimental tests for protrusion and undulation pressures in phospholipid bilayers, Biochem. 34, 8520-8532 (1995)

43. W. Helfrich: Steric interaction of fluid membranes in multilayer systems, Z. Naturforsch 33a 305-315 (1978)

44. C. R. Safinya, E. B. Sirota, D. Roux and G. S. Smith: Universality in interacting membranes: The effect of cosurfactants on the interfacial rigidity, Phys. Rev. Lett. 62, $1134(1989)$

45. R. R. Netz and R. Lipowsky: Stacks of fluid membranes under pressure and tension, Europhys. Lett. 29, 345-350 (1995)

46. D. Sornette and N. Ostrowsky: Importance of membrane fluidity on bilayer interactions, J. Chem. Phys. 84, 4062-4067 (1986)

47. R. Podgornik and V. A. Parsegian: Thermal-mechanical fluctuations of fluid membranes in confined geometries: The case of soft confinement, Langmuir $\mathbf{8}$, $577-562(1992)$

48. T. J. McIntosh and S. A. Simon: Contributions of hydration and steric (entropic) pressure to the interactions between phosphatidylcholine bilayers: Experiments with the subgel phase, Biochemistry 32, 8374-8384 (1993)

49. T. J. McIntosh and S. A. Simon: Hydration and steric pressures between phospholipid bilayers, Annu. Rev. Biophys. Biomol. Stuct. 23, 27-51 (1994).

50. H. I. Petrache, N. Gouliaev, S. Tristram-Nagle, R. Zhang, R. M. Suter and J. F. Nagle: Interbilayer interactions from high resolution X-ray scattering, Phys. Rev. E. 57, 7014-7024 (1998)

51. N. Gouliaev and J. F. Nagle: Simulations of a single membrane between two walls using a new Monte Carlo method, Phys. Rev. E 58, 881-888 (1998)

52. N. Gouliaev: Monte-Carlo simulations of membrane systems, CMU Doctoral Dissertation, 1998.

53. E. A. Evans and W. Rawicz: Entropy-driven tension and bending elasticity in condensed-fluid membranes, Phys. Rev. Lett. 64, 2094-2097 (1990)

54. P. Meleárd, C. Gerbeaud, T. Pott, L. Fernandez-Puente, I. Bivas, M. D. Mitov, J. Dufourcq and P. Bothorel: Bending elasticities of model membranes: Influence of temperature and sterol content, Biophys. J. 72, 2616 (1997)

55. V. A. Parsegian: Strength of van der Waals Attractions between Mica Surfaces across Lipid Bilayers, Langmuir 11, 1047 (1995) 